\title{
Scale Distributions and Fractal Dimensions in Turbulence
}

\author{
Haris J. Catrakis and Paul E. Dimotakis \\ Graduate Aeronautical Laboratories, California Institute of Technology, Pasadena, California 91125
}

(Received 18 March 1996; revised manuscript received 8 July 1996)

\begin{abstract}
A new geometric framework connecting scale distributions to coverage statistics is employed to analyze level sets arising in turbulence as well as in other phenomena. A 1D formalism is described and applied to Poisson, lognormal, and power-law statistics. A $d$-dimensional generalization is also presented. Level sets of $2 \mathrm{D}$ spatial measurements of jet-fluid concentration in turbulent jets are analyzed to compute scale distributions and fractal dimensions. Lognormal statistics are used to model the level sets at inner scales. The results are in accord with data from other turbulent flows. [S0031-9007(96)01540-2]
\end{abstract}

PACS numbers: 47.27.Ak, 02.50.-r, 47.10.+g, 47.53.+n

Many phenomena exhibit complex structure with a wide range of coexisting spatial and/or temporal scales participating in the dynamics [1], e.g., turbulent flow $[2,3]$, nephron cells [4], etc. The geometry of such phenomena can be quantified in terms of (constant) fractal dimensions, where power-law behavior is observed [1-4] or, in other cases, in terms of extensions of the fractal framework [5-11]. In turbulent mixing and combustion, in particular, such measures are useful for estimating the volume-fill fraction of isosurfaces of species composition. In this Letter, a new framework connecting coverage statistics to distributions of (various measures of) scales is applied to level sets in turbulence. Specifically, a 1D framework in terms of the probability density function of spacings formed by level crossings of signals is presented and applied to Poisson and lognormal statistics reported in $1 \mathrm{D}$ velocity and species-concentration measurements in various turbulent flows. Generalizations to multidimensional geometries are also introduced and applied to level sets of 2D spatial measurements of the jet-fluid concentration in turbulent jets. Lognormal statistics are used to model the level sets at small scales.

Dimensions of 1D point sets can be connected to the point-spacing probability density function (pdf) [10]. For homogeneous statistics, the fraction of contiguous $\lambda$ intervals required to cover the set, or coverage fraction $F_{1}(\lambda)$, can be identified as the geometric probability that a randomly located $\lambda$ interval contains part of the set. This can be expressed in terms of the (point) spacing pdf $p_{1}(l)$ as [10],

$$
\begin{aligned}
F_{1}(\lambda) & =\frac{1}{l_{m}} \int_{0}^{\lambda} \int_{\lambda^{\prime}}^{\infty} p_{1}(l) d l d \lambda^{\prime} ; \\
l_{m} & \equiv \int_{0}^{\infty} l p_{1}(l) d l,
\end{aligned}
$$

assuming a finite mean spacing $l_{m}$. Inverting,

$$
p_{1}(l)=-l_{m} \frac{d^{2} F_{1}(l)}{d l^{2}},
$$

as was previously derived for zero crossings of random Gaussian functions [12]. Denoting by $D_{1}(\lambda)$ the coverage (box-counting) dimension at a scale $\lambda$,

$$
D_{1}(\lambda) \equiv 1-\frac{d \ln F_{1}(\lambda)}{d \ln \lambda}=1-\frac{\lambda \int_{\lambda}^{\infty} p_{1}(l) d l}{\int_{0}^{\lambda} \int_{\lambda^{\prime}}^{\infty} p_{1}(l) d l d \lambda^{\prime}} .
$$

This can be viewed as a 1D (forward) transform between the dimension $D_{1}(\lambda)$ and the (point-spacing) scale distribution $p_{1}(l)$. This transform is invertible,

$$
\begin{aligned}
p_{1}(l)= & \frac{l_{m}}{l^{2}}\left\{D_{1}(l)\left[1-D_{1}(l)\right]+l \frac{d D_{1}(l)}{d l}\right\} \\
& \times \exp \left\{-\int_{l}^{\infty}\left[1-D_{1}\left(l^{\prime}\right)\right] \frac{d l^{\prime}}{l^{\prime}}\right\},
\end{aligned}
$$

where $l_{m}=\lim _{l \rightarrow 0}\left\{l \exp \left[\int_{l}^{\infty}\left[1-D_{1}\left(l^{\prime}\right)\right] d l^{\prime} / l^{\prime}\right]\right\}$, for a finite mean spacing [cf. Eq. (1)]. In the limit, $F_{1}(\lambda \rightarrow$ $0) \rightarrow \lambda / l_{m}$, i.e., the mean spacing determines the smallscale coverage fraction.

A first investigation of level crossings in turbulence was conducted by Liepmann [13], who measured the mean spacing of zero crossings in 1D velocity data. The corresponding spacing pdf was reported as described by Poisson statistics from measurements in turbulent boundary layers $[14,15]$, with constant fractal dimensions reported for such data [2]. For a Poisson point process, i.e., $p_{1}(l) d l=\exp \left(-l / l_{m}\right) d l / l_{m}$, however, the dimension is [cf. Eq. (3)]

$$
D_{1}(\lambda)=1-\frac{\lambda / l_{m}}{e^{\lambda / l_{m}}-1} .
$$

Figure 1 compares $D_{1}(\lambda)$, from Eq. (5), to ensembleaveraged estimates from five Monte Carlo simulations (standard deviation is smaller than symbol size in Figs. 1-3). Poisson-spacing records of length $L$, with $L / l_{m}=10^{3}$, were partitioned into contiguous $\lambda$ intervals and the number of intervals that cover the set was counted.

In various turbulent flows, level-crossing spacings derived from 1D scalar or velocity signals have been reported as well approximated by a lognormal pdf, 
as indicated for level crossings of 1D scalar measurements in turbulent jets [7] and in plumes dispersing in the atmospheric surface layer [16] as well as for zero crossings of $1 \mathrm{D}$ velocity measurements in turbu- lent boundary layers (in addition to Poisson fits) [14]. For a lognormal pdf, i.e., $p_{1}(l) d l=\exp \left\{-\left[\ln \left(l / l_{m}\right) /\right.\right.$ $\left.\sigma+\sigma / 2]^{2} / 2\right\} d l /(\sqrt{2 \pi} \sigma l)$, the dimension is [cf. Eq. (3)]

$$
D_{1}(\lambda)=1-\left\{1+\frac{l_{m}}{\lambda} \times\left[\frac{1+\operatorname{erf}\left[\left(\ln \left(\lambda / l_{m}\right) / \sigma-\sigma / 2\right) / \sqrt{2}\right]}{1-\operatorname{erf}\left[\left(\ln \left(\lambda / l_{m}\right) / \sigma+\sigma / 2\right) / \sqrt{2}\right]}\right]\right\}^{-1} .
$$

Figure 2 compares $D_{1}(\lambda)$, from Eq. (6), to five Monte Carlo simulations $\left(L / l_{m}=3 \times 10^{3}\right)$. This dimension also increases smoothly with scale, in accord with previous simulations [7].

Power-law statistics for $p_{1}(l)$, over a finite range of scales, e.g., $p_{1}(l) d l=a d l / l_{1}$, for $l<l_{1} ; a\left(l / l_{1}\right)^{-\nu} d l / l_{1}$, for $l_{1}<l<l_{2}$; and 0 , for $l_{2}<l$, correspond to a dimension plotted in Fig. 3, for $\nu=3 / 2$ and $l_{2} / l_{1}=10^{3}$. A comparison with five Monte Carlo simulations is also shown $\left(L / l_{m}=4 \times 10^{3}\right)$. In the limit of $l_{2} / l_{1} \gg 1$ and for scales $l_{1} \ll l \ll l_{2}$ [cf. Eq. (3)],

$$
D_{1}(\lambda) \rightarrow \nu-1 \text {, }
$$

for $1<\nu<2$ (cf. dashed line in Fig. 3 for

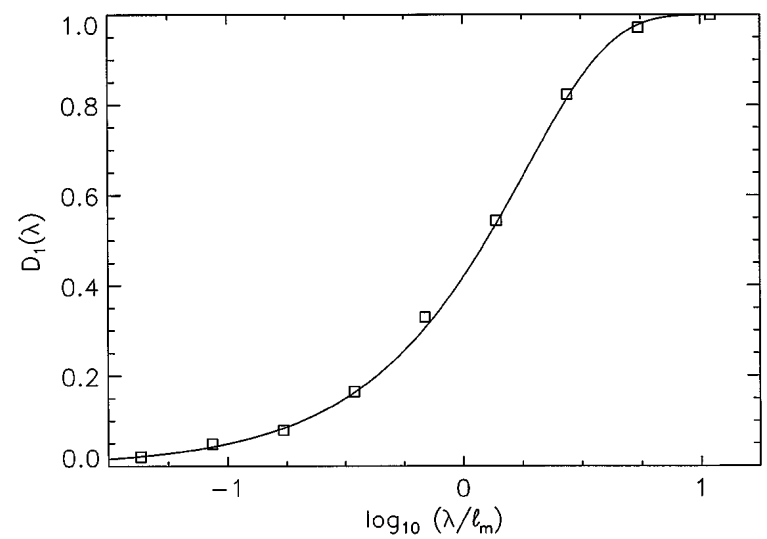

FIG. 1. Dimension as a function of scale for a Poisson point process. Theory: solid line, simulations: squares.

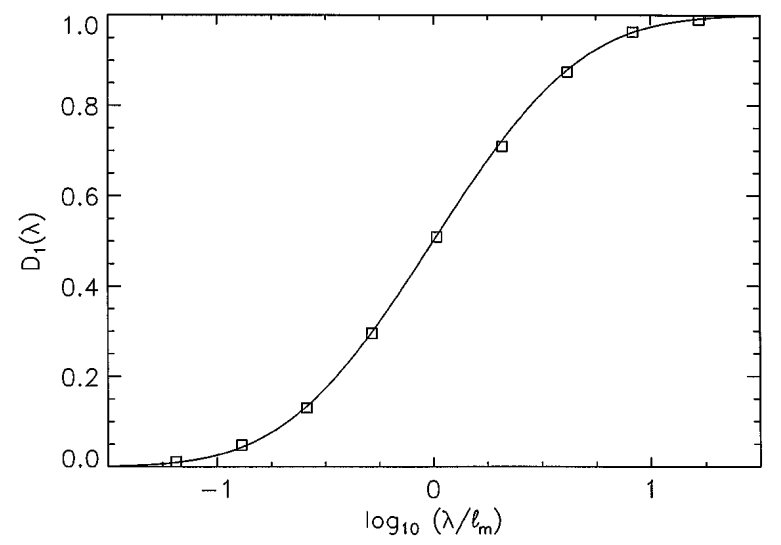

FIG. 2. $\quad D_{1}(\lambda)$ for a lognormal spacing pdf $(\sigma=1)$. Theory: solid line, simulations: squares. $\nu=3 / 2)$. Conversely, if $D_{1}(\lambda)=D_{1}=$ const., i.e. if $F_{1}(\lambda) \sim \lambda^{1-D_{1}}$, for $l_{1} \ll \lambda \ll l_{2}$,

$$
p_{1}(l) \sim l^{-D_{1}-1}
$$

[cf. Eq. (4)], i.e., the scale dependence of $D_{1}(\lambda)$, in this case, is a finite scale-range effect. A power-law coverage fraction, over a range of scales, implies a power-law $p_{1}(l)$ in the same range [cf. Eqs. (2) and (8)]. The converse, however, is not true-cf. the nonlocal nature of the forward transform [Eq. (1)].

For multidimensional geometries, an alternative scale measure is needed; the spacing scale cannot be extended to higher dimensions. Such a measure is the largestempty-interval scale (in 1D) defined as the size of the largest interval (centered) at a random location, that covers no part of the set [10]. The pdf of this scale $f_{1}(\lambda)$ is the probability (density) that a random location is a distance $\lambda / 2$ away from the nearest element of the set. It is given by [10]

$$
f_{1}(\lambda)=\frac{1}{l_{m}} \int_{\lambda}^{\infty} p_{1}(l) d l=\frac{d F_{1}(\lambda)}{d \lambda} .
$$

Generalizations to $d$ dimensions can be made. In terms of the coverage fraction $F_{d}(\lambda)$,

$$
f_{d}(\lambda) \equiv \frac{d F_{d}(\lambda)}{d \lambda}
$$

$f_{d}(\lambda)$ can be identified as the pdf of the largest-emptybox (LEB) scale, or size of the largest box, randomly located (centered), that contains no part of the set. For the

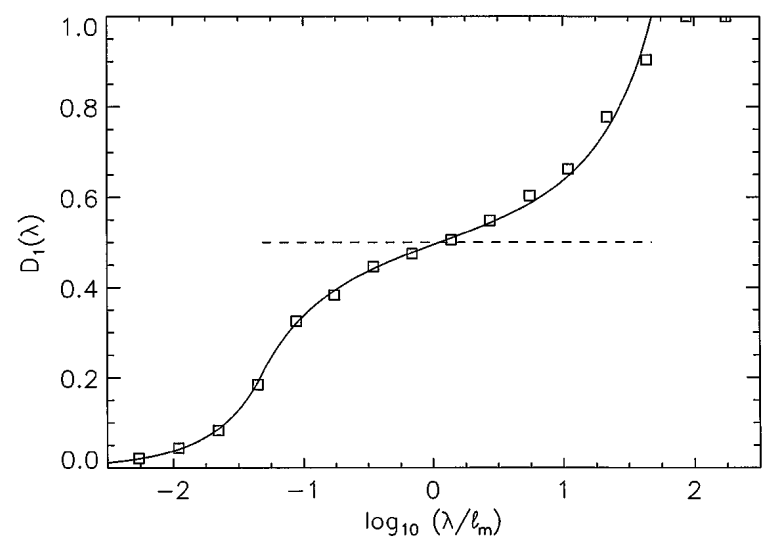

FIG. 3. $D_{1}(\lambda)$ for a power-law spacing pdf $\left(\nu=3 / 2, l_{2} / l_{1}=\right.$ $\left.10^{3}\right)$. Theory: solid line, simulations: squares. 


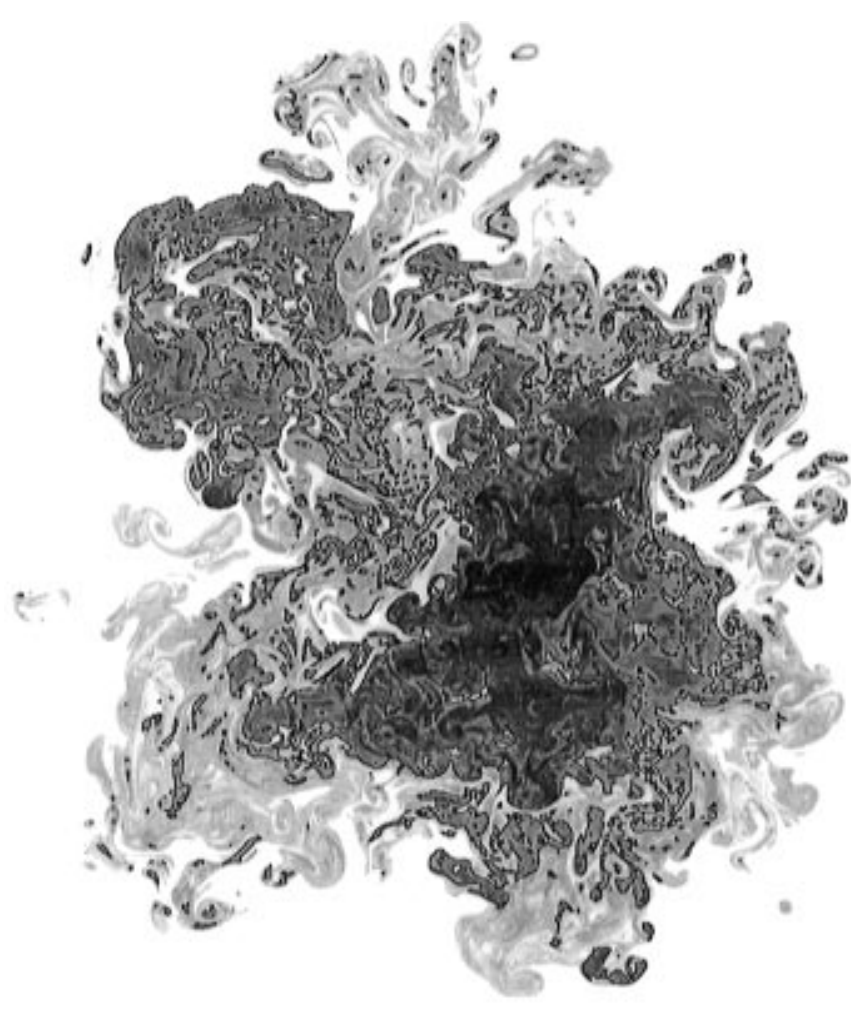

FIG. 4. Level set of jet-fluid concentration in the far field $\left(z / d_{j}=275\right)$ of a liquid-phase turbulent jet at $\operatorname{Re} \simeq 9 \times 10^{3}$.

dimension at a scale $\lambda$ and the LEB-scale pdf [cf. Eqs. (3) and (4)],

$$
\begin{aligned}
D_{d}(\lambda) & \equiv d-\frac{d \ln F_{d}(\lambda)}{d \ln \lambda}=d-\frac{\lambda f_{d}(\lambda)}{\int_{0}^{\lambda} f_{d}\left(\lambda^{\prime}\right) d \lambda^{\prime}}, \\
f_{d}(\lambda) & =\frac{d-D_{d}(\lambda)}{\lambda} \exp \left\{-\int_{\lambda}^{\infty}\left[d-D_{d}\left(\lambda^{\prime}\right)\right] \frac{d \lambda^{\prime}}{\lambda^{\prime}}\right\},
\end{aligned}
$$

are the forward and inverse $d$-dimensional transforms.

This framework can be used to compute the LEB-scale pdf for level sets derived from multidimensional measurements in turbulence, for example. Experiments were conducted to measure the jet-fluid concentration in the far field of liquid-phase turbulent jets for Reynolds numbers, $4.5 \times 10^{3} \leq \operatorname{Re} \leq 18 \times 10^{3}$, at a Schmidt number, $\mathrm{Sc} \simeq 1.9 \times 10^{3}$ [11]. Figure 4 depicts a level set of $2 \mathrm{D}$ spatial measurements of concentration at $\operatorname{Re} \simeq 9 \times 10^{3}$, recorded perpendicular to the jet axis $\left(z / d_{j}=275\right.$, where $d_{j}$ is the jet-nozzle diameter) using laser-induced fluorescence and digital-imaging techniques. This level set corresponds to the peak of the concentration pdf at this Re. The geometric complexity of such level sets is attributable, in part, to the large number $(\sim 700$, in each realization, on average) of islands and lakes, i.e., closed contours whose immediate interior is at higher or lower concentration, respectively, at this Reynolds number.
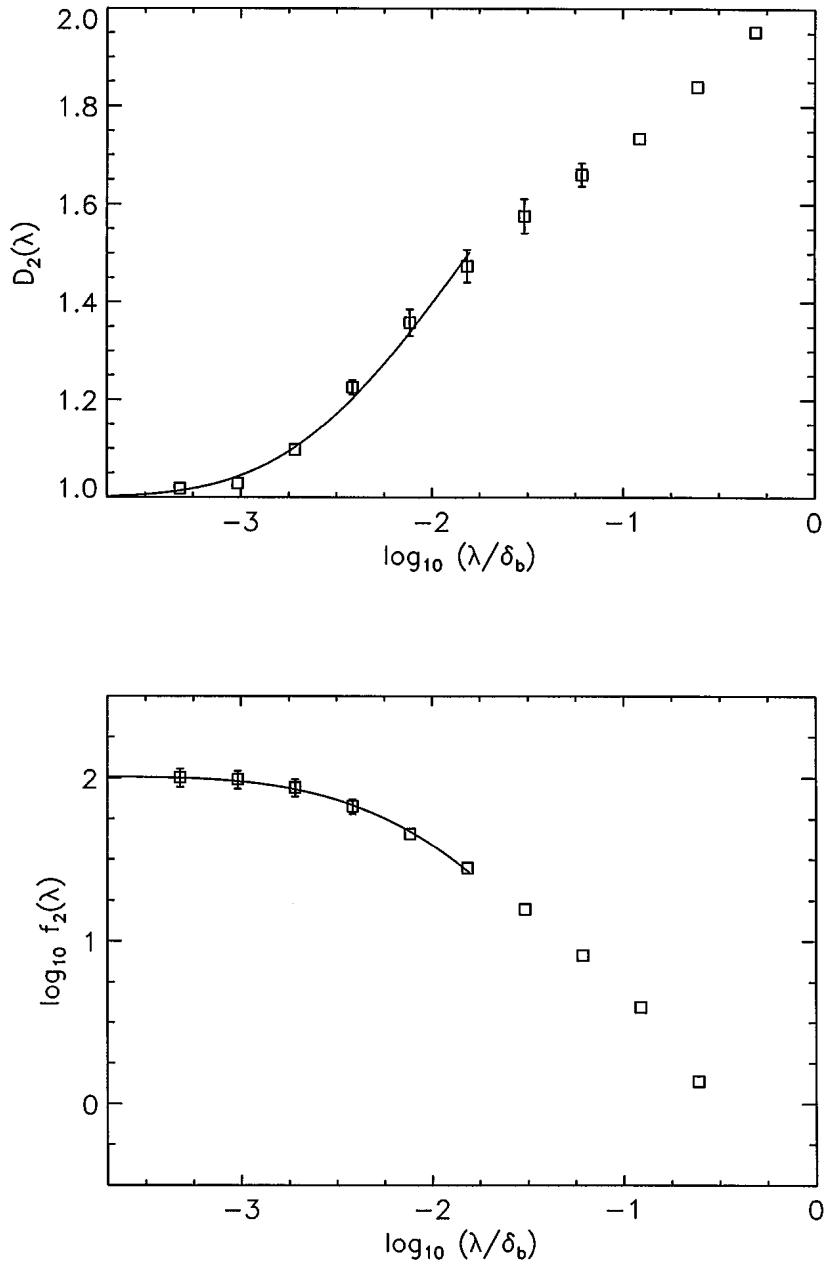

FIG. 5. Top: Dimension $D_{2}(\lambda)$ as a function of scale for level sets of concentration in a turbulent jet at $\mathrm{Re} \simeq 9 \times 10^{3}$. Bottom: Pdf of LEB scales $f_{2}(\lambda)$.

Figure 5 shows the ensemble-averaged dimension $D_{2}(\lambda)$ for level sets of concentration at $\operatorname{Re} \simeq 9 \times 10^{3}$ (six images) at a threshold corresponding to the peak of the concentration pdf. The smallest (diffusion) scale of the concentration field is estimated to be $\log _{10}\left(\lambda_{\mathcal{D}} / \delta_{b}\right) \simeq-3.0$, on the jet axis. For each level set, the $\delta_{b}$-sized bounding box was identified and partitioned into contiguous $\lambda$ boxes to compute the (coverage) fraction of the number of boxes that cover the level set, as a function of scale [11]. The dimension increases smoothly with the scale $\lambda$ and spans the full range of possible values for such data (error bars indicated if larger than symbol size).

Coverage statistics can now be used to compute the LEB-scale pdf (Fig. 5). While the jet is not (spatially) statistically homogeneous, $f_{2}(\lambda)$ retains its meaning, i.e., it is the pdf of the size of LEBs, randomly placed, interior to the $\delta_{b}$ box. The data indicate that the probability density of a LEB scale increases continuously with decreasing scale, tending to a constant as $\lambda \rightarrow 0$. 
At this stage, several models could be employed to fit the data in Fig. 5. Analysis of the size pdf of the islands/lakes, however, indicates lognormal statistics at inner scales (Fig. 6), where size, here, is defined as $A^{1 / 2}$, where $A$ is the area of each island/lake. Such statistics are consistent with fragmentation and growth (fission/ fusion) processes $[17,18]$. This finding suggests that lognormal statistics may be used to model the level set at inner scales. A 2D lognormal model [derived using the LEB-scale pdf computed for 1D lognormal spacings; cf. Eq. (6)] is shown in Fig. 5 at inner scales (solid lines) for the scale pdf (and for the dimension),

$$
f_{2}(\lambda) \propto \operatorname{erfc}\left[\left\{\ln \left(\lambda / l_{m}\right) / \sigma+\sigma / 2\right\} / \sqrt{2}\right] / 2 l_{m},
$$

with $\log _{10}\left(l_{m} / \delta_{b}\right) \simeq-1.5$ and $\sigma \simeq 1.2$, as fitted to the inner scales. The departure from the lognormal model, at large scales, indicates a break in behavior, suggesting that an alternate description at the outer scales of the flow is appropriate, as expected.

A useful interpretation of the LEB scale is as a measure of (twice) the distance to the nearest element of the level set. In turbulent combustion, for example, $f_{d}(\lambda)$ indicates the surface-to-volume (perimeter-to-area in 2D) ratio of surfaces (contours), located a distance $\lambda / 2$ (within a proportionality constant) from the instantaneous burning (level-set) surface. The LEB-scale pdf $f_{d}(\lambda)$ is, therefore, a scale-dependent measure of the surface-to-volume ratio, with the small-scale limit $f_{d}(\lambda \rightarrow 0)$ indicating the levelset surface-to-volume ratio.

In conclusion, the proposed framework can be used to compute the LEB-scale pdf from coverage statistics of complex, multidimensional geometries. Level sets arising

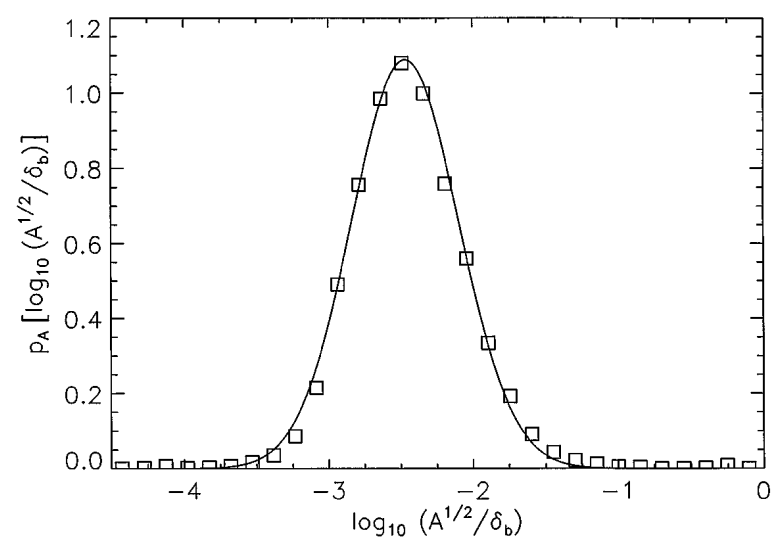

FIG. 6. Size pdf of islands and lakes at $\mathrm{Re} \simeq 9 \times 10^{3}$. in turbulence, for example, can be analyzed and modeled with this formalism. In turbulent jets, in particular, the LEB-scale pdf of level sets of concentration is consistent with a lognormal size pdf of islands/lakes, at the inner scales. Arguments for lognormal statistics have been put forth by Kolmogorov [17,19] and others [e.g., Ref. [18]] for stochastic fragmentation and growth processes, as may be expected to occur in turbulence, in general.

The support under AFOSR Grant No. F49260-94-10353 and discussions with C.L. Bond, M.C. Cross, A. Leonard, D. I. Pullin, and P. G. Saffman are gratefully acknowledged.

[1] B. B. Mandelbrot, The Fractal Geometry of Nature (W. H. Freeman \& Co., New York, 1982).

[2] K. R. Sreenivasan, Annu. Rev. Fluid Mech. 23, 539-600 (1991).

[3] P. Constantin, I. Procaccia, and K. R. Sreenivasan, Phys. Rev. Lett. 67, 1739-1742 (1991).

[4] D. Welling, J. Urani, L. Welling, and E. Wagner, Cell Physiol. 39, 953-963 (1996).

[5] H. Takayasu, J. Phys. Soc. Jpn. 51, 3057-3064 (1982).

[6] D. M. Mark and P. B. Aronson, Math. Geol. 16, 671-683 (1984).

[7] P.L. Miller and P.E. Dimotakis, Phys. Fluids A 3, 168-177 (1991).

[8] J.-P. Rigaut, in Fractals: Non-Integral Dimensions and Applications, edited by G. Cherbit (Wiley, Chichester, UK, 1991), pp. 151-187.

[9] P.E. Dimotakis, Nonlinear Sci. Today 2/91, 1, 27-31 (1991).

[10] H. J. Catrakis, Ph.D. thesis, California Institute of Technology, 1996.

[11] H. J. Catrakis and P.E. Dimotakis, J. Fluid Mech. 317, 369-406 (1996).

[12] M. S. Longuet-Higgins, Proc. R. Soc. London A 246, 99-118 (1958).

[13] H. W. Liepmann, Helv. Phys. Acta 22, 119 (1949).

[14] K. R. Sreenivasan, A. Prabhu, and R. Narasimha, J. Fluid Mech. 137, 251-272 (1983).

[15] P. Kailasnath and K. R. Sreenivasan, Phys. Fluids A 5, 2879-2885 (1993).

[16] E. Yee, R. Chan, P.R. Kosteniuk, G. M. Chandler, C. A. Biltoft, and J.F. Bowers, Bound. Layer Meteorol. 73, 53-90 (1995).

[17] A. N. Kolmogorov, Akad. Nauk SSSR 31, 99-101 (1941).

[18] R. E. Lopez, Mon. Weather Rev. 105, 865-872 (1977).

[19] A. N. Kolmogorov, J. Fluid Mech. 13, 82-85 (1962). 